\title{
Measurement of Lepton Flavour Violation at LHC with Long-Lived Slepton in the Coannihilation Region
}

\section{Takashi SHIMOMURA*†}

Departament de Física Teòrica and IFIC, Universitat de València - CSIC, E46100, Burjassot, València, Spain

E-mail: takashi.shimomuraeuv.es

Lepton Flavour Violation (LFV) of sleptons is studied in the Minimal Supersymmetric Standard Model (CMSSM) in the coannihilation scenario where the Lightest Supersymmetric Particle (LSP) is the neutralino, mostly Bino, and the Next to LSP (NLSP) is the lightest slepton, mostly stau. We show that lifetimes of such a long-lived slepton is sensitive to very small LFV parameters and can be measured at ATLAS deterctor in the LHC. Therefore, measurement of the lifetimes at LHC offers an excellent opportunity to investigate LFV in the slepton sector.

European Physical Society Europhysics Conference on High Energy Physics

July 16-22, 2009

Krakow, Poland

\footnotetext{
* Speaker.

$\dagger$ This work was supported in part by MEC and FEDER (EC), Grants No. FPA2005-01678 and the Generalitat Valenciana for support under the grants PROMETEO/2008/004, GV05/267 and GVPRE/2008/003.
} 


\section{Introduction}

Cosmological observations have confirmed the existence of non-baryonic dark matter. In a Minimal Supersymmetric Standard Model (MSSM) with conserved R-parity, the Lightest Supersymmetric Particle (LSP), usually the lightest neutralino, is stable and a perfect candidate for the dark matter. In fact, in the coannihilation region where the LSP neutralino and the Next to LSP (NLSP) slepton (stau) are degenerate, the observed relic abundance of the dark matter can be explained by that of the neutralino. When the mass difference between the neutralino and the slepton is smaller than the tau mass, the slepton can not decay into the neutralino and the tau, instead decay into the neutralino and the electron or muon through Lepton Flavour Violating (LFV) couplings. In this case, the lifetime of the slepton depends on the LFV couplings. In this proceeding, references and equations are omitted for the sake of limitation on pages but all of them can be found in [1].

\section{Lifetime of the Slepton}

In this section, the lifetimes of the slepton are shown for four values of the mass difference. We vary the mass insertion (MI), $\left(\delta_{R R}^{e}\right)_{e \tau}$ and $\left(\delta_{R R}^{e}\right)_{\mu \tau}$, defined in [1] to see the dependence and the sensitivity of the lifetime on the MIs.
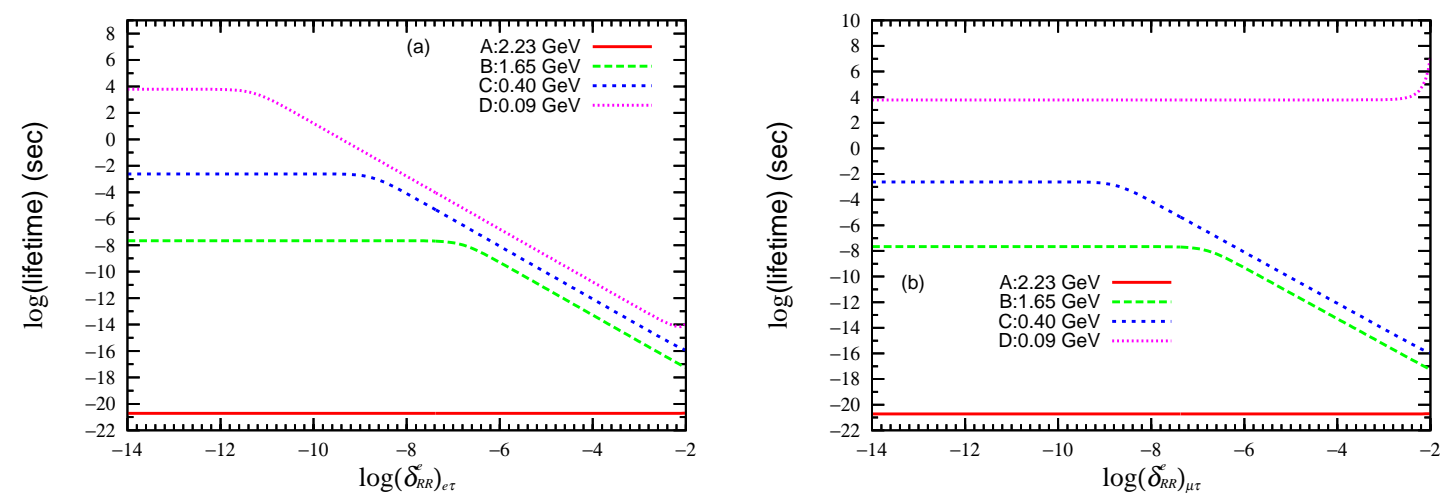

Figure 1: The lifetime of the lightest slepton as a function of $\delta_{R R}^{e}$. The left panel,(a), is the lifetime of the lightest slepton with the right-handed selectron and stau mixing, and the right panel, (b), is the one with the right-handed smuon and stau mixing. In both panels, the solid (red), dashed (green), dotted (blue), dotteddashed (pink) lines correspond to the mass difference, 2.23 (A), 1.65 (B), 0.40 (C), 0.09 (D), respectively.

In Fig. 1 (a), we show the lifetime of the lightest slepton, $\tau_{\tilde{l}_{1}}$, as a function of $\left(\delta_{R R}^{e}\right)_{e \tau}$. We vary it from $10^{-10}$ to $10^{-2}$. The mass difference for each curves is shown as $A, B, C$ and $D$ in the figures. We can see that the lifetime for $\delta m>m_{\tau}$ does not change, because the decay of slepton to neutralino and tau is always dominant and the lifetime is insensitive to $\left(\delta_{R R}^{e}\right)_{e \tau}\left(\lesssim 10^{-2}\right)$. On the other hand, for $\delta m<m_{\tau}$, the lifetime grows more than 13 orders of magnitude in the limit $\left(\delta_{R R}^{e}\right)_{e \tau} \rightarrow 0$, where the three- or four-body decays are dominant. With this mass difference, the two-body decay into $\tau$ and $\tilde{\chi}_{1}^{0}$ is forbidden but those into $e$ (or $\mu$ for $\left(\delta_{R R}^{e}\right)_{\mu \tau} \neq 0$ ) and $\tilde{\chi}_{1}^{0}$ are allowed through LFV couplings. The lifetime decreases proportionally to $\left|\left(\delta_{R R}^{e}\right)_{e \tau}\right|^{-2}$ when the two-body decay dominates the total decay width. From these figures, we can see that the lifetime 
is sensitive to $\delta^{e} \mathrm{~S}$ up to $\simeq 10^{-7}-10^{-11}$. Comparing the present bounds on the MIs given in [1], above values of $\delta^{e}$ s are much smaller and can not be reached by experiments as $\tau \rightarrow \mu$ (or $e$ ) $\gamma$, etc. Fig. 1) (b) is a plot in the case of $\left(\delta_{R R}^{e}\right)_{\mu \tau}$. The lifetime is constant for $\delta m=0.09 \mathrm{GeV}$ to $\left(\delta_{R R}^{e}\right)_{\mu \tau}$ because the two-body decay into muon is forbidden. For larger $\delta m$, we observe again the same dependence on $\left(\delta_{R R}^{e}\right)_{\mu \tau}$. The same figures for the left-handed mixing is given in [1].

\section{LHC Phenomenology}

In this section, we discuss a expected phenomenology of the long-lived slepton scenario at the LHC experiment, focusing mainly on the ATLAS detector.

We start with estimating the number of the lightest sleptons, $\tilde{l}_{1}$, produced at LHC. Based on the post-WMAP SUSY benchmark point $J^{\prime}$ proposed in [2] which is close to our spectrum with a slightly different $\tan \beta$ value, the total branching ratios for $\tilde{l}_{1}$ production are 0.86 in $\tilde{q}_{L}$ decay, $0.72 / 0.90$ for $\tilde{t}_{1} / \tilde{t}_{2}$ and $0.87 / 0.67$ for $\tilde{b}_{1} / \tilde{b}_{2}$ at $\tan \beta=35$. The total cross section of SUSY pair production is given as $\sigma_{\mathrm{SUSY}}=130 \mathrm{fb}$ in [3]. Assuming the integrated luminosity, $\mathscr{L}_{\text {int }}=30 \mathrm{fb}^{-1}$, the number of the lightest sleptons produced, $N_{\tilde{l}_{1}}$, is estimated as $N_{\tilde{l}_{1}} \simeq 4290$. Next, we estimate the Lorentz factor $\beta \gamma$. The slepton mass in our analysis is $290-320 \mathrm{GeV}$ and the typical momentum of outgoing sleptons is expected to be $500-900 \mathrm{GeV}$, hence $\beta \gamma$ is $1.53 \lesssim \beta \gamma \lesssim 2.75$. Here we assume $\beta \gamma=2$ for simplicity. Then, using $N_{\tilde{l}_{1}}$ and $\beta \gamma$, we show the expected number of slepton decays within each detector layer in Table 1 . As can be seen, when the lifetime, $\tau_{\tilde{l}_{1}}$, is below $10^{-9}$ sec., most of the sleptons decay inside the pixel detector, while almost half of them decay inside the inner detector and nearly all of them decay within the detector when $\tau_{\tilde{l}_{1}} \sim 10^{-8}$ sec. If $\tau_{\tilde{l}_{1}}$ is between $10^{-8}$ and $10^{-6} \mathrm{sec}$., several hundreds of slepton decays occur inside the ATLAS detector. Almost all of them escape from the detector when $\tau_{\tilde{l}_{1}}>10^{-5}$ sec., although we expect order of 10 decays inside the detector for $\tau_{\tilde{l}_{1}} \simeq 10^{-5} \mathrm{sec}$.

Table 1: The expected number of slepton decays in the ATLAS detector. The length is minimum $(5 \mathrm{~cm})$ and medium $(50 \mathrm{~cm})$ distance to the pixel detector and the maximum distance to the outer boundary of the detectors from the interaction point corresponding to pixel detector $(3.1 \mathrm{~m})$, calorimeter $(5.8 \mathrm{~m})$ and muon spectrometer $(25.0 \mathrm{~m})$. The lifetime is varied from $10^{-11}$ to $10^{-5}$ seconds.

\begin{tabular}{|c|c|c|c|c|c|}
\hline & $5 \mathrm{~cm}$ & $50 \mathrm{~cm}$ & $3.1 \mathrm{~m}$ & $5.8 \mathrm{~m}$ & $25.0 \mathrm{~m}$ \\
\hline $10^{-5} \mathrm{sec}$. & 0.04 & 0.36 & 2.2 & 4.1 & 17.8 \\
$10^{-6} \mathrm{sec}$. & 0.36 & 3.6 & 22.1 & 41.3 & 175.1 \\
$10^{-7} \mathrm{sec}$. & 3.6 & 35.6 & 216.0 & 395.3 & 1461.9 \\
$10^{-8} \mathrm{sec}$. & 35.6 & 343.0 & 1731.0 & 2658.3 & 4223.5 \\
$10^{-9} \mathrm{sec}$. & 343.0 & 2425.6 & 4265.5 & 4289.7 & 4290.0 \\
$10^{-10} \mathrm{sec}$. & 2425.6 & 4289.0 & 4290.0 & 4290.0 & 4290.0 \\
$10^{-11} \mathrm{sec}$. & 4289.0 & 4290.0 & 4290.0 & 4290.0 & 4290.0 \\
\hline
\end{tabular}

Sleptons with different lifetimes would give different signatures in the ATLAS detector. For $\tau_{\tilde{l}_{1}}<10^{-11} \mathrm{sec}$, since almost all of the sleptons would decay before they reach the first layer of the pixel detector, we would not observe any heavy charged-particle track in the detector. In this case, it would be more difficult to identify the presence of long-lived sleptons at the ATLAS detector. 
For $\tau_{\tilde{l}_{1}} \sim 10^{-10}$ to $10^{-9} \mathrm{sec}$, almost all the sleptons would decay inside the pixel detector and leave a charged track with a kink. A light charged-particle would cross the outer detectors and a corresponding track and/or hit would be seen in each detector. Thus, by combining signals, we could identify whether the outgoing charged-particle is an electron or muon. Therefore, if we can fix the mass difference between the lightest slepton and the neutralino, we can determine the value of the MI from Figs. 1. In the case of right-handed slepton mixing, the lifetimes between $10^{-10}$ and $10^{-8} \mathrm{sec}$. would correspond to $\left(\delta_{R R}^{e}\right)_{e \tau}$ between $10^{-7}$ and $10^{-4}$ with the mass difference, $m_{e}<\delta m<m_{\tau}$, and $\left(\delta_{R R}^{e}\right)_{\mu \tau}$ between $10^{-7}$ and $10^{-5}$ with $m_{\mu}<\delta m<m_{\tau}$. Similarly, in the case of left-handed slepton mixing, the same lifetimes would correspond to $\left(\delta_{L L}^{e}\right)_{e \tau}$ between $4 \times 10^{-6}$ and $10^{-3}$ with $m_{e}<\delta m<m_{\tau}$, and $\left(\delta_{L L}^{e}\right)_{\mu \tau}$ between $4 \times 10^{-6}$ and $10^{-4}$ with $m_{\mu}<\delta m<m_{\tau}$. For $\tau_{\tilde{l}_{1}} \sim$ $10^{-8}$ sec., about half of sleptons would decay inside the inner detector and the rest would decay inside the calorimeters and/or muon spectrometer. In this case, it would be important to determine whether the decay is LFV two-body decay or lepton flavour conserving three-body decay. For lifetimes between $10^{-7}$ and $10^{-5} \mathrm{sec}$., very few sleptons would decay inside the pixel detector and most of them would escape the detector leaving a charged track with a corresponding hit in muon spectrometer. Even for particles escaping the detector, we could use muon spectrometer to determine slepton mass and momentum as studied in [4]. For $\tau_{\tilde{l}_{1}} \gtrsim 10^{-5}$ sec., a very few sleptons would decay inside the ATLAS detector. In this case, we can put lower bound on the lifetime.

\section{Summary}

We have shown the dependence of the lightest slepton lifetimes on different mass insertions, $\left(\delta_{R R / L L}^{e}\right)_{e \tau}, \mu \tau$ for values of $\delta m$ from 2.23 to $0.09 \mathrm{GeV}$. We found that the lifetimes are proportional to $\left|\delta^{e}\right|^{-2}$ until three- or four-body decays become comparable to the LFV decays. By comparing the values of the bounds on $\delta^{e} \mathrm{~s}$, we can see that the lifetimes are sensitive to much smaller values of these $\delta^{e}$ s, even to future sensitivities of proposed experiments. We have also discussed the expected phenomenology at the ATLAS detector. We have estimated the number of slepton decays in the different detectors, assuming an integrated luminosity $\mathscr{L}_{\text {int }}=30 \mathrm{fb}^{-1}$ and $\beta \gamma=2$ (Table. 11). We have seen that the ATLAS detector can observe lifetimes in the range of $10^{-11}$ to $10^{-6}$ sec., and these lifetime would correspond to $\left(\delta_{R R}^{e}\right)_{e \tau, \mu \tau}$ between $10^{-7}$ and $10^{-3}$ and $\left(\delta_{L L}^{e}\right)_{e \tau, \mu \tau}$ between $4 \times 10^{-6}$ and $10^{-3}$. Therefore the LHC offers a very good opportunity to study lepton flavour violation.

\section{References}

[1] Kaneko S, Sato J, Shimomura T, Vives O and Yamanaka M, Phys. Rev. D 78, 116013 (2008) [arXiv:0811.0703 [hep-ph]].

[2] Battaglia M, De Roeck A, Ellis J R, Gianotti F, Olive K A and Pape L, Eur. Phys. J. C 33, 273 (2004) [arXiv:hep-ph/0306219].

[3] Skands P Z, Eur. Phys. J. C 23, 173 (2002) [arXiv:hep-ph/0110137].

[4] Tarem S et al. ATL-PHYS-PUB-2005-02 